\title{
Photometry and low resolution spectroscopy of hot post-AGB candidates
}

\author{
G. Gauba ${ }^{1}$, M. Parthasarathy ${ }^{1}$, Brijesh Kumar ${ }^{2}$, R. K. S. Yadav ${ }^{2}$, and R. Sagar ${ }^{1,2}$ \\ 1 Indian Institute of Astrophysics, Koramangala, Bangalore 560034, India \\ 2 State Observatory, Manora Peak, Nainital 263129 (Uttaranchal), India
}

Received 5 June 2002 / Accepted 7 March 2003

\begin{abstract}
We have obtained Johnson $U, B, V$ and Cousins $R, I$ photometry and low resolution spectra of a small sample of hot post-AGB candidates. Using the present data in combination with $J H K$ data from 2MASS, infrared data from the MSX catalog and the IRAS fluxes, we have studied the spectral energy distribution (SED) of these stars. Using the DUSTY code we have estimated the dust temperatures, the distances to the stars, the mass-loss rates, angular radii of the inner boundary of the dust envelopes and dynamical ages from the tip of the AGB. These candidates have also been imaged through a narrow band $\mathrm{H} \alpha$ filter, to search for nebulosity around the central stars. Our $\mathrm{H} \alpha$ images revealed the bipolar morphology of the low excitation PN IRAS 17395-0841 with an angular extent of 2.8". The bipolar lobes of IRAS 17423-1755 in H $\alpha$ were found to have an angular extent of 3.5" (south-east lobe) and 2.2" (north-west lobe). The dust envelope characteristics, low resolution spectrum and IRAS colors suggest that IRAS 18313-1738 is similar to the proto-planetary nebula (PPN) HD 51585. The SED of IRAS 17423-1755, IRAS 18313-1738 and IRAS 19127+1717 show a warm dust component (in addition to the cold dust) which may be due to recent and ongoing mass-loss.
\end{abstract}

Key words. stars: AGB and post-AGB - stars: early-type - stars: evolution - stars: mass-loss - stars: circumstellar matter

\section{Introduction}

Stars having initial mass between 0.8 and $8 M_{\odot}$ pass through the asymptotic giant branch (AGB) phase of evolution (Iben \& Renzini 1983). These low and intermediate mass stars, undergo severe mass-loss $\left(10^{-7}-10^{-4} M_{\odot} \mathrm{yr}^{-1}\right)$ on the AGB. The postAGB phase is the rapid evolution of the star from the tip of the AGB to the planetary nebula (PN) stage. During the postAGB phase the circumstellar dust shell moves away from the star and decreases in temperature from $\sim 400$ to $100 \mathrm{~K}$ (Bedijn 1987; Zijlstra et al. 1992). The rapidly evolving, hotter analogues (OB spectral types) of the cooler (G, F, A) post-AGB stars (Parthasarathy \& Pottasch 1986, 1989) are interesting to study in order to understand the mass-loss mechanisms, dust envelope characteristics and dynamical time scales responsible for the evolution of low and intermediate mass stars to PNe of varied morphologies. In this paper, we analyse the complete spectral energy distribution of a small sample of hot post-AGB candidates. We have also searched for nebulosity around these stars. As part of our search for new hot post-AGB candidates, we also present the spectra of two high galactic latitude early B-type supergiants.

Send offprint requests to: G. Gauba, e-mail: gauba@iiap.ernet.in

\section{Target selection}

Hot post-AGB stars may be identified on the basis of their high temperature, low surface gravity, evidence of remnant AGB envelope (infrared excess), detached dust envelope, double peaked spectral energy distribution and nebulosity due to scattered light (Parthasarathy et al. 2000a; Kwok 2001). Pottasch et al. (1988) and van der Veen \& Habing (1988) identified a region of the IRAS color-color diagram $(F(12 \mu) / F(25 \mu)<0.35$ and $F(25 \mu) / F(60 \mu)>0.3)$ which was mainly populated by stars in transition from the AGB to the PN phase. An occasional HII region, Seyfert galaxy, or T Tau star is not excluded from this range (Pottasch et al. 1988; Szczerba et al. 2001). But in the case of post-AGB stars, the circumstellar dust temperatures are in the range of 100-200 K (Kwok 2001). In addition to the cold dust, warm dust indicative of recent or ongoing post-AGB mass loss may also be present in the circumstellar envelopes of these stars (e.g. Hen401, Parthasarathy et al. 2001). Young massive OB supergiants are not expected at high galactic latitudes. Also young massive OB supergiants do not have detached cold circumstellar dust shells. High galactic latitude OB supergiants with detached dust shells and far-infrared colors similar to $\mathrm{PNe}$ were indeed found to be in the post-AGB stage of evolution (Parthasarathy 1993a; Parthasarathy et al. 2000a). To firmly establish the evolutionary status it is important to obtain high resolution optical spectra of the candidate stars for determination 
Table 1. List of hot post-AGB candidates selected for photometry.

\begin{tabular}{|c|c|c|c|c|c|c|c|c|}
\hline \hline IRAS & Name & $l$ & $b$ & Sp. Type & \multicolumn{4}{|c|}{ IRAS Fluxes (Jansky) } \\
& & & & & $12 \mu$ & $25 \mu$ & $60 \mu$ & $100 \mu$ \\
\hline $17074-1845$ & Hen 3-1347 & 4.1 & +12.3 & B3IIIe & 0.50 & 12.20 & 5.66 & $3.47:$ \\
$17395-0841$ & SS 318 & 17.0 & +11.1 & PN & 0.31 & 4.18 & 8.43 & 6.38 \\
$17423-1755$ & Hen 3-1475 & 9.4 & +5.8 & Be & 7.05 & 28.31 & 63.68 & 33.43 \\
$18237-0715$ & MWC 930 & 23.6 & +2.2 & Be & 1.84 & 4.01 & 38.24 & 35.13 \\
$18313-1738$ & MWC 939 & 15.3 & -4.3 & Be & 9.41 & 7.28 & $1.00:$ & $68.70 \mathrm{~L}$ \\
$19127+1717$ & SS 438 & 51.0 & +2.8 & B9V & 12.15 & 18.79 & 8.50 & $7.37 \mathrm{~L}$ \\
$19157-0247$ & LS IV -0229 & 33.6 & -7.2 & B1III & 8.88 & 7.16 & 2.45 & $11.05 \mathrm{~L}$ \\
$19200+3457$ & StHA161 & 67.6 & +9.5 & B & $0.25 \mathrm{~L}$ & 2.12 & 1.45 & $1.41 \mathrm{~L}$ \\
$19399+2312$ & LS II +2317 & 59.3 & +0.1 & B1III & 1.13 & $2.27:$ & $23.31 \mathrm{~L}$ & $80.20 \mathrm{~L}$ \\
\hline
\end{tabular}

A colon: indicates moderate quality IRAS flux, $\mathrm{L}$ is for an upper limit.

Table 2. Characteristics of the hot post-AGB candidates selected spectroscopically.

\begin{tabular}{ccccccccc}
\hline \hline Object & RA & Dec & Sp. & $V$ & $E(B-V)$ & $l$ & $b$ & $d(\mathrm{kpc})$ \\
& $(2000)$ & $(2000)$ & Type & & & & & \\
\hline LSE 163 $=$ & $13: 08: 46$ & $-43: 27: 51$ & B2 I & 10.4 & 0.1 & 306.3 & +19.3 & 4.3 \\
CD-42 8141 & & & & & & & & \\
\hline LSE 45 & $13: 49: 18$ & $-50: 22: 46$ & B2 I & 11.0 & 0.2 & 312.3 & +11.4 & 4.8 \\
CD-49 8217 & & & & & & & & \\
\hline
\end{tabular}

of their chemical abundances and to look for signatures of AGB nucleosynthesis.

The targets selected for photometry (Table 1) were IRAS sources with $\mathrm{OB}$ spectral types having $F(12 \mu) / F(25 \mu)<0.35$ and $F(25 \mu) / F(60 \mu)>0.3$. Based on their low resolution spectra and spectral type, many of these targets had been classified as post-AGB stars by Parthasarathy et al. (2000a). Drilling $\&$ Bergeron (1995) extended the survey of luminous stars in the Milky Way to galactic latitude $b= \pm 30^{\circ}$ for $l= \pm 60^{\circ}$. They designated this as the LSE survey. We have also selected few LSE stars at high galactic latitudes with OB-spectral types (Table 2). The optical counterparts of the IRAS sources were carefully identified using the Digitised Sky Survey and NASA's Sky View Java interface. The targets were searched within the IRAS error boxes. Besides most of the targets are in scarcely populated regions at high galactic latitudes.

\section{Observations}

$U, B, V, R, I$ images of the hot post-AGB candidates were obtained on 10 April, 2000 at the State Observatory (SO) with the $f / 13$ Cassegrain $104 \mathrm{~cm}$. Sampurnanand Carl-Zeiss telescope. The central $1280 \times 1280$ pixels of a $2 \mathrm{~K} \times 2 \mathrm{~K} \mathrm{CCD}$ were used for the purpose, in $2 \times 2$ binning mode, resulting in $680 \times 680$ pixels image frames. The PG 1323-086 standard stars field (Landolt 1992) was observed several times during the night for calibration and extinction measurements.

The narrow band images of the stars were obtained with the same telescope on 11 April, 2000 with a $1 \mathrm{~K} \times 1 \mathrm{~K} \mathrm{CCD}$ having plate scale of $0.37^{\prime \prime}$ pixel $^{-1}$. An $\mathrm{H} \alpha$ filter centered at $6565 \AA$ with a bandwith of $80 \AA$ and a continuum filter centered at $6650 \AA$ with a bandwith of $80 \AA$ were used for the purpose.
Optical spectra of the hot post-AGB candidates were obtained with the $1.02 \mathrm{~m}$ telescope at the Vainu Bappu Observatory (VBO) on 20 January, 2000 with a resolution of $1.1 \AA$ pixel $^{-1}$. Appropriate number of flat fields and bias frames were observed on each night. Following each observation of a program star, we obtained a comparison lamp spectra and the spectra of a bright star close to the RA and Dec of the object.

\section{Analysis of photometric and spectroscopic data}

The DAOPHOT package in IRAF was used for the reduction and analysis of the photometric data (Massey \& Davis 1992). Bias subtraction and flat fielding was performed on the individual image frames. The extinction coefficient for each filter was determined from the standard star observations. The observed photometric magnitudes of the objects are listed in Table 3a. The 2MASS (2Micron All Sky Survey) Catalog was searched within $15^{\prime \prime}$ of each object for their JHK magnitudes. Whenever possible, the $J H K$ magnitudes have been taken from García-Lario et al. (1997). We also searched the MSX (Midcourse Space Experiment) catalog within 3" of the objects. The MSX fluxes are listed in Table 3b. The spectral types of the objects have been taken from literature.

The objects were imaged through the $\mathrm{H} \alpha$ and continuum filters. To detect $\mathrm{H} \alpha$ emission, we followed the procedure outlined by Beaulieu et al. (1999). The continuum images were scaled to match the total star signal above the sky in their corresponding $\mathrm{H} \alpha$ images. An appropriate offset was then applied to each continuum image to equate the sky level to that of the $\mathrm{H} \alpha$ image. To find the $\mathrm{H} \alpha$ emitting objects, we then divided the $\mathrm{H} \alpha$ image by the continuum image for each field. We detected $\mathrm{H} \alpha$ emission in four of the objects. Figure 1 shows the continuum and resultant $\mathrm{H} \alpha$ images of the objects. 
Table 3a. Photometry of hot post-AGB candidates.

\begin{tabular}{|c|c|c|c|c|c|c|c|c|c|}
\hline $\begin{array}{l}\text { IRAS } \\
\text { name }\end{array}$ & $\begin{array}{c}U \\
\mathrm{mag}\end{array}$ & $\begin{array}{c}B \\
\text { mag }\end{array}$ & $\begin{array}{c}V \\
\text { mag }\end{array}$ & $\begin{array}{c}R \\
\text { mag }\end{array}$ & $\begin{array}{c}I \\
\mathrm{mag}\end{array}$ & $\begin{array}{c}J \\
\mathrm{mag} \\
\end{array}$ & $\begin{array}{c}H \\
\text { mag } \\
\end{array}$ & $\begin{array}{c}K \\
\text { mag } \\
\end{array}$ & $\begin{array}{c}\mathrm{H}_{\alpha} \\
\text { emission }\end{array}$ \\
\hline $17074-1845$ & - & $11.92^{1}$ & 11.55 & 11.25 & 11.07 & $\begin{array}{c}11.33^{2} \pm \\
0.13\end{array}$ & $\begin{array}{c}12.06^{2} \pm \\
0.16\end{array}$ & $\begin{array}{c}11.02^{2} \pm \\
0.11\end{array}$ & $\mathrm{NO}$ \\
\hline $17395-0841$ & 15.37 & 15.04 & 13.74 & 12.79 & 11.94 & $\begin{array}{c}10.59^{2} \pm \\
0.06\end{array}$ & $\begin{array}{c}9.76^{2} \pm \\
0.03\end{array}$ & $\begin{array}{c}9.21^{2} \pm \\
0.04\end{array}$ & YES \\
\hline $17423-1755$ & 13.57 & $13.3^{3}$ & 12.64 & 11.75 & 10.91 & $\begin{array}{c}9.61^{2} \pm \\
0.05\end{array}$ & $\begin{array}{c}8.32^{2} \pm \\
0.02 \\
\end{array}$ & $\begin{array}{c}6.80^{2} \pm \\
0.02 \\
\end{array}$ & YES \\
\hline $18237-0715$ & 15.48 & 14.88 & 12.37 & 10.51 & 8.78 & - & - & - & YES \\
\hline $18313-1738$ & 12.36 & 12.88 & 12.37 & 11.60 & 11.37 & $\begin{array}{l}9.995 \pm \\
0.027 \\
\end{array}$ & $\begin{array}{c}8.374 \pm \\
0.045 \\
\end{array}$ & $\begin{array}{c}6.785 \pm \\
0.019 \\
\end{array}$ & YES \\
\hline $19127+1717$ & 14.70 & 14.20 & 13.13 & 12.38 & 8.65 & $11.07^{4}$ & $9.94^{4}$ & $8.65^{4}$ & $\mathrm{NO}$ \\
\hline $19157-0247$ & 11.62 & 11.31 & 10.55 & 10.04 & 9.48 & - & - & - & $\mathrm{NO}$ \\
\hline $19200+3457$ & 10.59 & 11.35 & 11.25 & 11.14 & 11.09 & $\begin{array}{c}11.008 \pm \\
0.024\end{array}$ & $\begin{array}{c}10.883 \pm \\
0.025\end{array}$ & $\begin{array}{c}10.739 \pm \\
0.032\end{array}$ & $\mathrm{NO}$ \\
\hline $19399+2312$ & - & 10.90 & 10.05 & 9.46 & 8.85 & $\begin{array}{c}8.573 \pm \\
0.022\end{array}$ & $\begin{array}{c}8.388 \pm \\
0.003\end{array}$ & $\begin{array}{c}8.331 \pm \\
0.033\end{array}$ & NO \\
\hline
\end{tabular}

${ }^{1}$ The $B$ magnitude of IRAS17074-1845 is from the Tycho-2 Catalog (Hog et al. 2000).

${ }^{2}$ From Garcia-Larío et al. (1997).

${ }^{3}$ The B magnitude of IRAS17423-1755 is from the USNO-A2.0 Catalog (Monet et al. 1998).

${ }^{4}$ From Whitelock \& Menzies (1986).

${ }^{5}$ The rms errors in $U, B, V, R, I$ obtained from fitting the transformation equations to the standard stars are $\pm 0.05, \pm 0.05, \pm 0.05, \pm 0.09, \pm 0.07$ respectively.

Table 3b. MSX data.

\begin{tabular}{|c|c|c|c|c|}
\hline \hline \multirow{2}{*}{ IRAS } & \multicolumn{4}{|c|}{ MSX Fluxes (Jansky) } \\
\cline { 2 - 5 } & Band $A$ & Band $C$ & Band $D$ & Band $E$ \\
& $8.28 \mu$ & $12.13 \mu$ & $14.65 \mu$ & $21.34 \mu$ \\
\hline $18313-1738$ & 8.9623 & 7.5263 & 6.0883 & 8.3414 \\
$19127+1717$ & 8.332 & 11.737 & 12.577 & 16.636 \\
\hline
\end{tabular}

The optical spectra were bias subtracted, flat field corrected, wavelength calibrated and continuum normalised using the IRAF package. Figure 2 shows the continuum normalised spectra from $4250 \AA$ to $5500 \AA$. Spectral classification was performed using the spectra of standard stars from Jacoby et al. (1984).

\subsection{Central star temperatures and reddening}

Whenever, the spectral type and luminosity class of the star was known, the effective temperature of the central star, was taken from Lang (1992). For Be stars and for the bipolar PPN Hen3-1475, we assumed a central star temperature of $20000 \mathrm{~K}$. The total extinction (I.S.+C.S.) towards the stars due to interstellar and circumstellar dust grains was estimated from the difference between the observed and intrinsic $(B-V)$ values. For Be stars, we assumed intrinsic $(B-V)=-0.20$ corresponding to an effective temperature of $20000 \mathrm{~K}$ and for the low excitation PN IRAS 17395-0841 we assumed intrinsic $(B-V)=-0.3$ corresponding to an effective temperature of $35000 \mathrm{~K}$ for the hot central star. Since the distances to the stars were not known, the interstellar extinction (I.S.) in the direction of the stars was estimated using the Diffuse Infrared Background Experiment (DIRBE)/IRAS dust maps (Schlegel et al. 1998). The DIRBE/IRAS reddening estimates have an accuracy of $16 \%$. The difference between the two sets of values would give an estimate of the extinction due to circumstellar dust. The DIRBE/IRAS dust maps do not give reliable estimates of the interstellar extinction for $|b|<5^{\circ}$. The $U, B, V, R, I$ and near infrared magnitudes of the stars were corrected for the total extinction $(E(B-V)$ I.S.+C.S.) assuming $R_{v}=3.1$.

\subsection{Modelling of the circumstellar dust envelopes with DUSTY code}

To derive physical parameters from the spectral energy distribution of the stars, we solved the problem of radiation transfer through a dust envelope using the DUSTY code (Ivezić et al. 1999) developed at the University of Kentucky assuming centrally-heated spherical density distributions. The central stars were assumed to be point sources, at the center of the spherical density distributions and their spectral energy distributions were taken to be Planckian. For modelling, we chose from six different grain types: "warm" (Sil-Ow) and "cold" (Sil-Oc) silicates from Ossenkopff et al. (1992), silicates and graphites (Sil-Dl and grf-DL) from Draine \& Lee (1984), amorphous carbon (amC-Hn) from Hanner (1988) and $\mathrm{SiC}$ (SiC-Pg) from Pégourié (1988). DUSTY contains data for the optical properties of these six grain types. For all stars except 

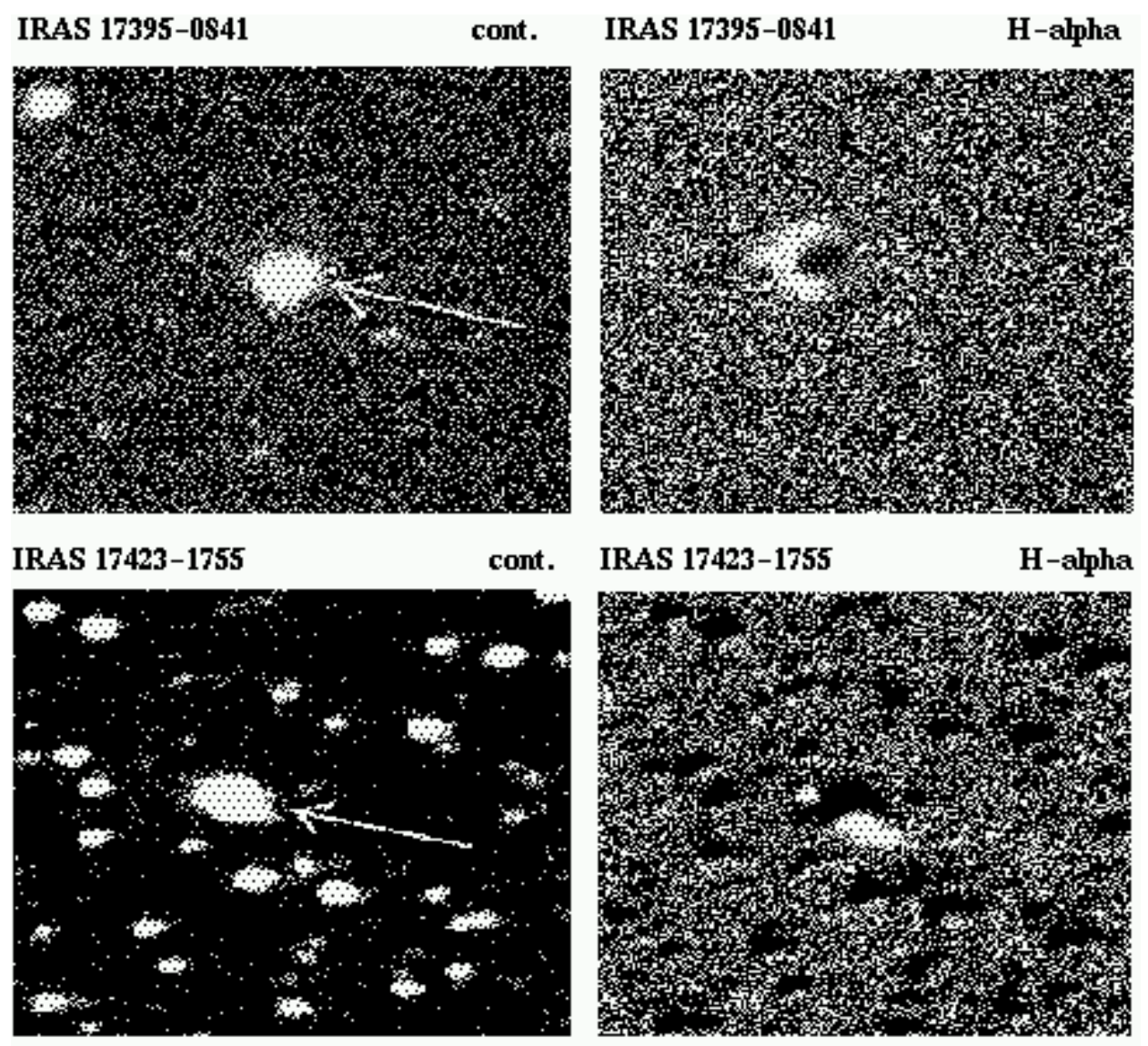

IRAS 18237-0715

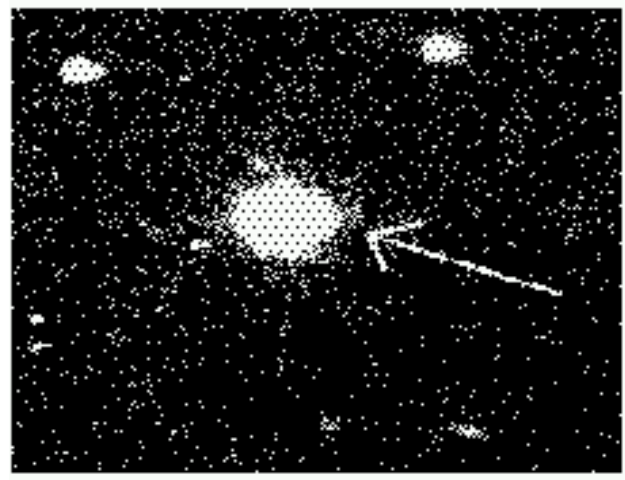

cont. IRAS 18237-0715

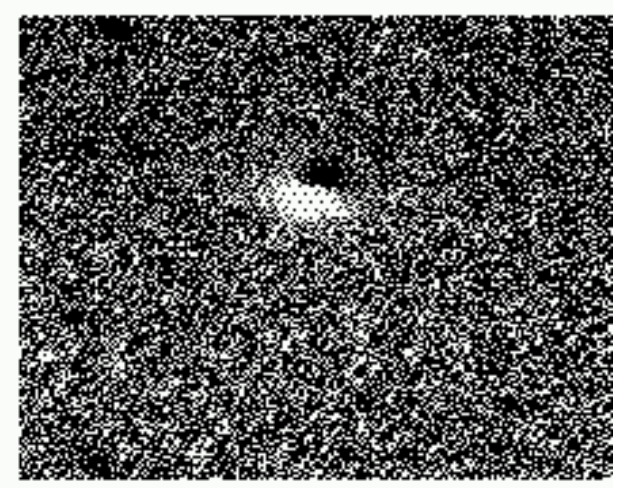

cont. IRAS 18313-173s

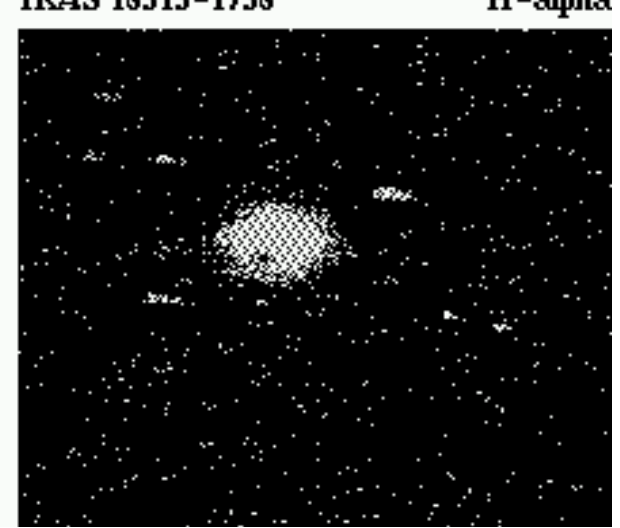

Fig. 1. Continuum ( $\lambda=6650 \AA, \Delta \lambda=80 \AA)$ and $\mathrm{H} \alpha(\lambda=6565 \AA, \Delta \lambda=80 \AA)$ images of the selected hot post-AGB stars. 

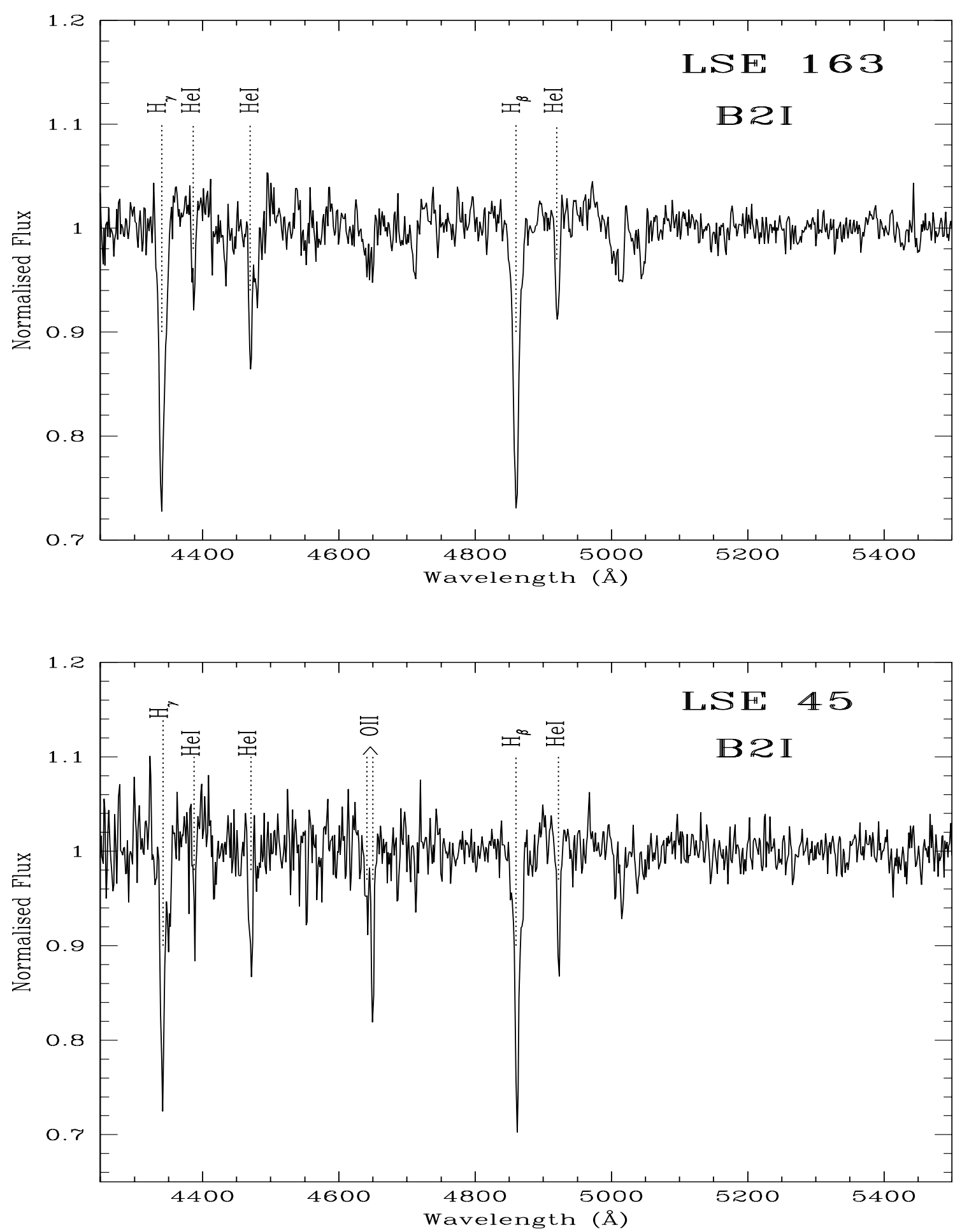

Fig. 2. Low resolution spectra of LSE 163 and LSE 45.

IRAS 17395-0841 and MWC930 the standard Mathis, Rumpl, Nordsieck (MRN) (Mathis et al. 1977) power-law was used for the grain size $(n(a))$ distributions, i.e. $n(a) \propto a^{-q}$ for $a_{\min } \leq$ $a \leq a_{\max }$ with $q=3.5, a(\min )=0.005 \mu$ and $a(\max )=0.25 \mu$. For IRAS 17395-0841 and MWC930, we used the MRN distribution with $q=4.3, a(\min )=0.005 \mu, a(\max )=0.25 \mu$ and $q=3.7, a(\min )=0.10 \mu$ and $a(\max )=0.25 \mu$ respectively. The dust temperature $\left(T_{\mathrm{d}}\right)$ on the inner shell boundary and the optical depth $(\tau)$ at $0.55 \mu$ were varied assuming an inverse square law $\left(y^{-2}\right)$ for the spherical density distribution. The shell was assumed to extend to 1000 times its inner radius. For MWC930 and LSII+2317, we had to adopt a different power law $\left(y^{-p}\right)$ for the density distribution in the shell with $p=1.3$ and 0.55 respectively inorder to obtain a good fit. DUSTY does not allow simultaneous modelling of warm and cold dust shells. Hence, the cold dust in the case of Hen3-1475, MWC939 and SS438 had to be modelled and treated independent of the warm dust in these stars. We adopted the fits for which the sum of squares of 
Table 4. Input physical parameters for DUSTY and the adopted reddening values.

\begin{tabular}{ccccccc}
\hline \hline IRAS & dust & $E(B-V)$ & $E(B-V)$ & $T_{*}$ & grain & Optical \\
& type & C.S.+I.S. & I.S. & $(\mathrm{K})$ & Sil-DL & amC-Hn \\
\hline $17074-1845$ & cold & 0.66 & 0.28 & 17100 & amC-Hn & 0.105 \\
\hline $17395-0841$ & cold & 1.60 & 1.23 & 35000 & SiC-Pg & 0.16 \\
\hline $17423-1755$ & warm & 0.86 & 0.67 & 20000 & SiC-Pg & 0.35 \\
\hline $18237-0715$ & cold & 0.86 & 0.67 & 20000 & grf-DL & 0.004 \\
\hline $18313-1738$ & warm & 0.71 & - & 20000 & Sil-DL & 0.2 \\
& cold & 0.71 & - & 20000 & grf-DL & 0.16 \\
\hline $19127+1717$ & warm & 1.14 & - & 10500 & SiC-Pg & 0.07 \\
& cold & 1.14 & - & 10500 & Sil-DL, grf-DL \& amC-Hn & 0.7 \\
\hline $19157-0247$ & cold & 1.02 & 0.65 & 24000 & Sil-Ow & 0.004 \\
\hline $19200+3457$ & cold & 0.30 & 0.14 & 20000 & SiC-Pg & 0.04 \\
\hline $19399+2312$ & cold & 1.11 & - & 24000 & & \\
\hline
\end{tabular}

the deviations between the observed and modelled fluxes (after scaling) were a minimum. Table 4 lists the adopted input parameters. Figure 3 shows the spectral energy distribution of the stars.

Having fixed $T_{\mathrm{d}}$ and $\tau$, we then used the gas-dynamical mode of the DUSTY code, to derive the mass-loss rate for a given model. However, in the case of MWC930 and LSII+2317 where the density distribution follows a different law, a hydrodynamics calculation and hence the determination of mass-loss rate was not possible. The output of the code gives the shell inner radius, $r 1(\mathrm{~cm})$ where the dust temperature $\left(T_{\mathrm{d}}\right)$ is specified. The radius scales in proportion to $L^{1 / 2}$ where $L$ is the luminosity and the output value corresponds to $L=10^{4} L_{\odot}$. The mass-loss rate $(\dot{M})$ scales in proportion to $L^{3 / 4}\left(r_{\mathrm{gd}} \rho_{\mathrm{s}}\right)^{1 / 2}$ where, the gas-to-dust mass ratio, $r_{\mathrm{gd}}=200$ and the dust grain density, $\rho_{\mathrm{s}}=3 \mathrm{~g} \mathrm{~cm}^{-3}$. We carried out calculations for hot postAGB stars with core masses of $0.565 M_{\odot}$ and $0.605 M_{\odot}$ corresponding to luminosities of $4500 L_{\odot}$ (Schönberner 1983) and $6300 L_{\odot}$ (Blöcker 1995) respectively. The distances $(d)$ were derived using $r 1$ and the ratio of the observed and modelled fluxes at $0.55 \mu$.

$\theta(=r 1 / d)$ is the angular radii of the inner boundary of the cold circumstellar dust envelopes. In some cases e.g. IRAS $19399+2312, \theta$ is very large $\left(16^{\prime \prime}\right)$ but the nebula is not detected in the optical and in the case of IRAS 18237-0715, $\theta=45^{\prime \prime}$ but the detected nebulosity is less than $2^{\prime \prime}$. In these cases, the scattered light radiated by the dust shells in the optical may be too faint to be seen by our observations. In the $V$-band, some of our stars have a flux of the order of $6.9 \times 10^{-11} \mathrm{erg} \mathrm{s}^{-1} \mathrm{~cm}^{-2}$, e.g. IRAS $17395-0841(V=13.74)$. The reflection nebular (scattered light) flux per pixel on the CCD frames will be several orders of magnitude fainter. Our maximum exposure in $\mathrm{H} \alpha$ was 5 min which is not sufficient enough to reach our telescope's detection limit in $\mathrm{H} \alpha$. As we move away from the central star, the scattered light flux drops down rapidly (see e.g. the radial intensity profile of AFGL 2688 in Kwok et al. 2001). Longer exposures would be required to detect the fainter reflection nebulae. High resolution HST images (e.g. Su et al. 2001) and subarcsecond mid-infrared imaging (Kwok et al. 2002) have been used to resolve PPNe.

The time when the star leaves the AGB can be assumed to coincide with the ejection of its dust envelope. For post-AGB stars with core masses of $0.565 M_{\odot}$ and $0.605 M_{\odot}$ the effective temperatures at the tip of the AGB are $5000 \mathrm{~K}$ (Schönberner $1983)$ and $6000 \mathrm{~K}$ (Blöcker 1995) respectively. The dust condensation temperature is taken to be $1200 \mathrm{~K}$ (Whittet 2003). Using the hydrodynamic mode of the DUSTY code, we then obtained the terminal outflow velocities $\left(V_{\mathrm{e}}\right)$ and the distances $(r 0)$ at which the dust grains reached the condensation temperature. $V_{\mathrm{e}}$ scales in proportion to $L^{1 / 4}\left(r_{\mathrm{gd}} \rho_{\mathrm{s}}\right)^{-1 / 2}$. The present inner boundary of the dust envelopes is at $r 1$. Thus, the dynamical age from the tip of the AGB is given by $(r 1-r 0) / V_{\mathrm{e}}$. The $V_{\mathrm{e}}$ values are in good agreement with the expansion velocity of $10 \mathrm{~km} \mathrm{~s}^{-1}$ assumed by Volk \& Kwok (1989) for models of PPNe and the expansion velocities for PPNe estimated from CO measurements by Loup et al. (1990). There is an inherent uncertainity of $30 \%$ in the $(\dot{M})$ and $V_{\text {e }}$ values obtained with the DUSTY code (Ivezić et al. 1999).

Tables $5 \mathrm{a}$ and $\mathrm{b}$ list the respective values for $T_{\mathrm{d}}, r 1, r 0, d, \theta$, $\dot{M}, V_{\mathrm{e}}$ and $\Delta t$. All calculations were carried out using the best fit parameters for the cold circumstellar dust shells.

\subsection{The LSE stars}

On comparing the low resolution spectra of the high galactic latitude stars, LSE163 and LSE45 with that of standard stars from Jacoby et al. (1984) we classified them as B2I. They may be similar to PG 1323-086 and PG 1704+222, two high galactic latitude B-type post-AGB stars (Moehler \& Heber 1998). Using the relation between core-mass and quiescent luminosity maximum for AGB stars (Wood \& Zaro 1981), with a typical core-mass of $0.6 M_{\odot}$, we estimated a value of $10^{3.79} L_{\odot}$ for the luminosity of the central stars. Since, $M_{\text {bol }}(\operatorname{Sun})=4.75$, we obtained an absolute bolometric magnitude of -4.73 for the stars. Applying the bolometric correction for the spectral type of the 
Table 5a. Derived stellar and dust envelope parameters for $M_{\mathrm{c}}=0.565$.

\begin{tabular}{ccccccccc}
\hline \hline IRAS & $\begin{array}{c}T_{\mathrm{d}} \\
(\mathrm{K})\end{array}$ & $\begin{array}{c}r 1 \\
(\mathrm{~cm})\end{array}$ & $\begin{array}{c}r 0 \\
(\mathrm{~cm})\end{array}$ & $\begin{array}{c}d \\
(\mathrm{kpc})\end{array}$ & $\begin{array}{c}\theta \\
\left({ }^{\prime \prime}\right)\end{array}$ & $\begin{array}{c}\dot{M} \\
M_{\odot} \mathrm{yr}^{-1}\end{array}$ & $\begin{array}{c}V_{\mathrm{e}} \\
\mathrm{km} \mathrm{s}^{-1}\end{array}$ & $\begin{array}{c}\Delta t \\
\mathrm{yr}\end{array}$ \\
\hline $17074-1845$ & 122 & $3.9 \times 10^{16}$ & $1.1 \times 10^{14}$ & 3.1 & 0.8 & $1.0 \times 10^{-5}$ & 17.4 & 743 \\
\hline $17395-0841$ & 110 & $1.7 \times 10^{17}$ & $1.4 \times 10^{14}$ & 1.0 & 11.7 & $1.1 \times 10^{-6}$ & 7.7 & 7344 \\
\hline $17423-1755$ & 100 & $2.9 \times 10^{17}$ & $1.5 \times 10^{14}$ & 3.1 & 6.3 & $5.3 \times 10^{-5}$ & 23.3 & 4154 \\
\hline $18237-0715$ & 100 & $1.4 \times 10^{17}$ & - & 0.2 & 45.0 & - & - & - \\
\hline $18313-1738$ & 450 & $2.3 \times 10^{15}$ & $1.1 \times 10^{14}$ & 3.6 & 0.04 & $3.6 \times 10^{-6}$ & 18.9 & 39 \\
\hline $19127+1717$ & 300 & $6.2 \times 10^{15}$ & $1.4 \times 10^{14}$ & 4.0 & 0.1 & $9.0 \times 10^{-6}$ & 25.9 & 78 \\
\hline $19157-0247$ & 135 & $1.1 \times 10^{17}$ & $1.4 \times 10^{14}$ & 0.8 & 9.1 & $1.4 \times 10^{-6}$ & 8.8 & 4180 \\
\hline $19200+3457$ & 140 & $3.1 \times 10^{16}$ & $1.5 \times 10^{14}$ & 3.8 & 0.5 & $4.0 \times 10^{-6}$ & 12.8 & 805 \\
\hline $19399+2312$ & 127 & $1.3 \times 10^{17}$ & - & 0.6 & 16.0 & - & - & - \\
\hline
\end{tabular}

Table 5b. Derived stellar and dust envelope parameters for $M_{\mathrm{c}}=0.605$.

\begin{tabular}{ccccccccc}
\hline \hline IRAS & $\begin{array}{c}T_{\mathrm{d}} \\
(\mathrm{K})\end{array}$ & $\begin{array}{c}r 1 \\
(\mathrm{~cm})\end{array}$ & $\begin{array}{c}r 0 \\
(\mathrm{~cm})\end{array}$ & $\begin{array}{c}d \\
(\mathrm{kpc})\end{array}$ & $\begin{array}{c}\theta \\
\left({ }^{\prime \prime}\right)\end{array}$ & $\begin{array}{c}\dot{M} \\
M_{\odot} \mathrm{yr}^{-1}\end{array}$ & $\begin{array}{c}V_{\mathrm{e}} \\
\mathrm{km} \mathrm{s}^{-1}\end{array}$ & $\begin{array}{c}\Delta t \\
\mathrm{yr}\end{array}$ \\
\hline $17074-1845$ & 122 & $4.6 \times 10^{16}$ & $1.8 \times 10^{14}$ & 3.7 & 0.8 & $1.3 \times 10^{-5}$ & 20.6 & 743 \\
\hline $17395-0841$ & 110 & $2.0 \times 10^{17}$ & $2.3 \times 10^{14}$ & 1.1 & 11.8 & $1.5 \times 10^{-6}$ & 8.8 & 7543 \\
\hline $17423-1755$ & 100 & $3.5 \times 10^{17}$ & $2.6 \times 10^{14}$ & 3.7 & 6.3 & $6.8 \times 10^{-5}$ & 26.0 & 4481 \\
\hline $18237-0715$ & 100 & $1.7 \times 10^{17}$ & - & 0.2 & 45.0 & - & - & - \\
\hline $18313-1738$ & 450 & $2.7 \times 10^{15}$ & $1.9 \times 10^{14}$ & 4.2 & 0.04 & $4.7 \times 10^{-6}$ & 22.3 & 38 \\
\hline $19127+1717$ & 300 & $7.4 \times 10^{15}$ & $2.3 \times 10^{14}$ & 4.7 & 0.1 & $1.2 \times 10^{-5}$ & 28.7 & 84 \\
\hline $19157-0247$ & 135 & $1.3 \times 10^{17}$ & $2.4 \times 10^{14}$ & 0.9 & 9.2 & $1.8 \times 10^{-6}$ & 9.9 & 4336 \\
\hline $19200+3457$ & 140 & $3.7 \times 10^{16}$ & $2.5 \times 10^{14}$ & 4.6 & 0.5 & $5.1 \times 10^{-6}$ & 14.7 & 834 \\
\hline $19399+2312$ & 127 & $1.6 \times 10^{17}$ & - & 0.7 & 16.0 & - & - & - \\
\hline
\end{tabular}

star (Lang 1992), and using the distance modulus method, we obtained distance estimates of 4.3 and $4.8 \mathrm{kpc}$ respectively to these stars. If on the other hand, we adopt the absolute visual magnitude $\left(M_{\mathrm{v}}\right)$ of -6.4 for a normal Population I B-type supergiant we obtain distance estimates of 19.3 and $21.7 \mathrm{kpc}$ respectively. Such large distance estimates, suggesting that these objects lie at the outer edge of our galaxy appear to be unphysical. Abundance analysis from the high resolution spectra of these objects may confirm their evolutionary status as evolved low mass post-AGB stars.

\section{Notes on individual objects}

\section{\#IRAS 17074-1845 (=Hen3-1347)}

Henize (1976) had identified it as an $\mathrm{H} \alpha$ emission line object. It was classified as a hot post-AGB star on the basis of its high galactic latitude, far-infrared colors similar to PNe and Be spectral type (Parthasarathy 1993b). Based on near-IR observations, García-Lario et al. (1997) also classified it as a post-AGB star. Parthasarathy et al. (2000a) classified it as a B3IIIe post-AGB star. They found $\mathrm{H} \beta$ and $\mathrm{H} \gamma$ in emission. From our $\mathrm{H} \alpha$ image of this object, we did not detect nebulosity around the central star. The angular radius of the envelope inner boundary indicates a compact nebula $\left(<2^{\prime \prime}\right)$. The spectral type, dust temperature, double peaked spectral energy distribution and mass-loss rate indicate that it may be a post-AGB star evolving into the PN stage.

\#IRAS 17395-0841 (=SS 318)

It was discovered as a young low excitation planetary nebula by Vijapurkar et al. (1997). The $\mathrm{H} \alpha$ image (300 s exposure) of the PN is clearly extended. Figure 4 shows a contour plot of the object in $\mathrm{H} \alpha$. The angular extent of the nebula at the FWHM of the $\mathrm{H} \alpha$ image is $2.8^{\prime \prime}$. It appears that bipolar outflows are just beginning to form in this nebula, similar to the case of Hen3-1357 (Bobrowsky et al. 1998). High resolution $\mathrm{H} \alpha$ images with longer exposure times may reveal the true morphology of this young PN.

\#IRAS17423-1755 (=Hen3-1475)

On the basis of IRAS data, Parthasarathy \& Pottasch (1989) first classified it as a hot post-AGB star. It is a bipolar protoplanetary nebula (PPN) (Bobrowsky et al. 1995; Riera et al. 1995). The collimated bipolar outflows, seen in our $\mathrm{H} \alpha$ image have been studied by Borkowski et al. (1997) and Bobrowsky et al. (1995). Wind velocities greater than $1000 \mathrm{~km} \mathrm{~s}^{-1}$ have been observed in this PPN (Sanchez Contreras \& Sahai 2001; Borkowski \& Harrington 2001). From our $\mathrm{H} \alpha$ image, the south-east lobe appears to be more extended with an angular extent of $3.5^{\prime \prime}$. The north-west lobe has an angular extent of only $2.2^{\prime \prime}$. Based on [NII] HST images of the object, Borkowski et al. (1997) concluded that Hen3-1475 is a point symmetric nebula. They found emission knots in the outflows 


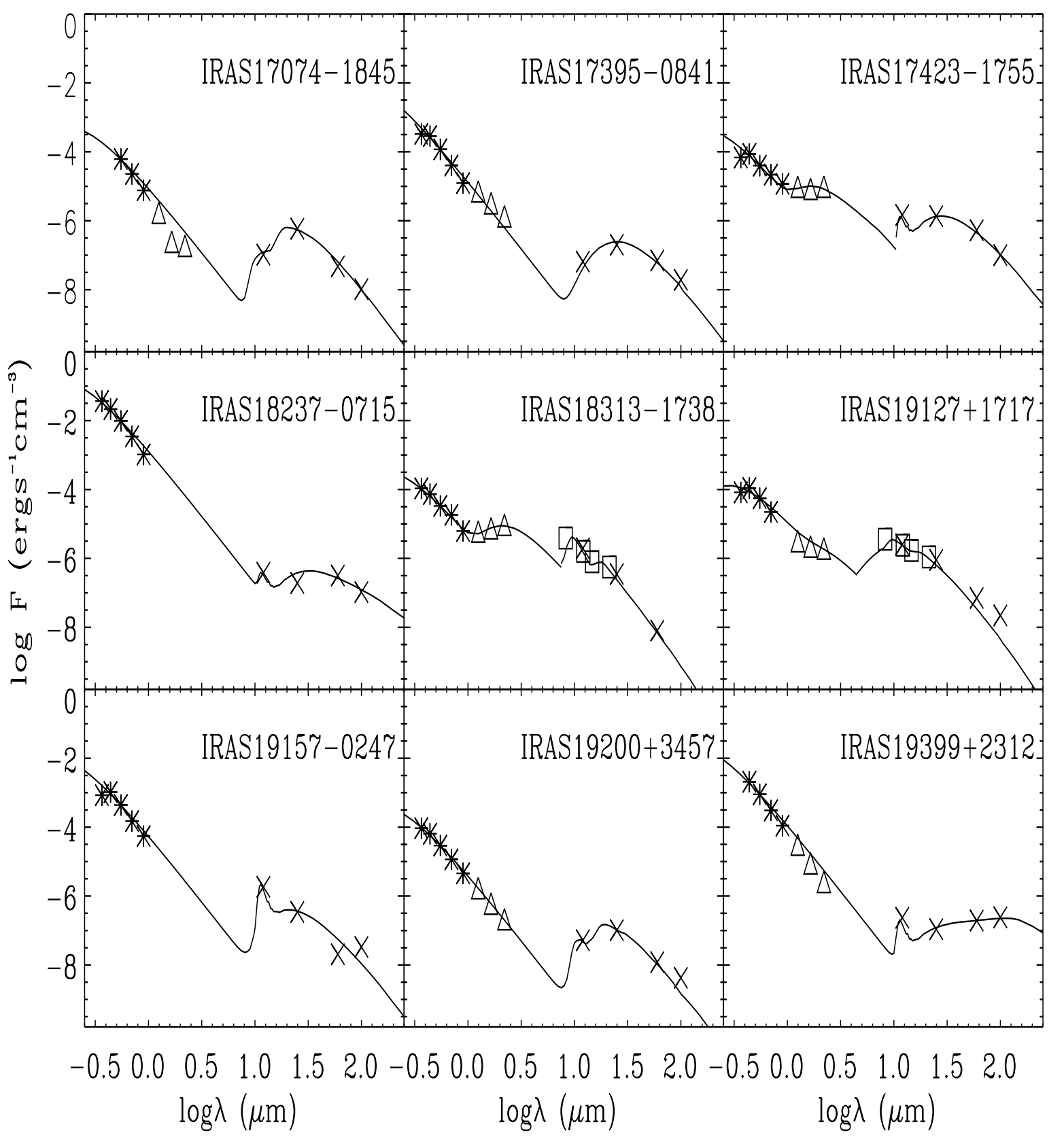

Fig. 3. Spectral Energy distributions (SED) of the hot post-AGB candidates. UBVRI data (asterisk) are plotted alongwith $J H K$ (triangle), MSX (square) and IRAS data (cross). DUSTY model fits are shown by solid lines.

perpendicular to the dusty torus surrounding the central star with the outermost pair of knots at 7.82" and 7.57" from the star. From the spectral energy distribution, we detected a warm dust component at $1500 \mathrm{~K}$ indicating cirumstellar dust close to the central star as a result of ongoing post-AGB mass-loss. The dynamical age from the tip of the AGB was estimated to be $\sim 4000 \mathrm{yrs}$. This value is uncertain due to the limitations of the DUSTY code and its inability to simultaneously model the warm and cold circumstellar dust (see Discussion below).

\#IRAS 18237-0715(=MWC930)

Its spectra (Parthasarathy et al. 2000a; Vijapurkar et al. 1998) showed Balmer lines and several permitted and forbidden lines of [FeII] in emission. We found strong $\mathrm{H} \alpha$ emission in this star indicating the presence of a low excitation PN with an angular extent of less than $2^{\prime \prime}$. Our seeing ( 2 arcsec) limited images could not resolve the nebula. There is considerable extinction $(E(B-V)=2.1)$ towards the star. At a distance of only $0.2 \mathrm{kpc}$, the heavy obscuration due to dust may be responsible for our inability to resolve the nebula. The effective temperature of the central star, spectral energy distribution and $\mathrm{H} \alpha$ emission suggest that it may be a hot post-AGB star. High resolution spectra and images of this object may help in understanding its true nature.

\#IRAS 18313-1738(=MWC939)

Based on strong FeII and [FeII] emission lines Allen \& Swings (1976) classified it as a Be star. In the spectrum taken in April, 1994 with the $1 \mathrm{~m}$ CTIO telescope, covering the wavelength range from $3800 \AA$ to $5020 \AA$ at a resolution of $2 \AA$, 


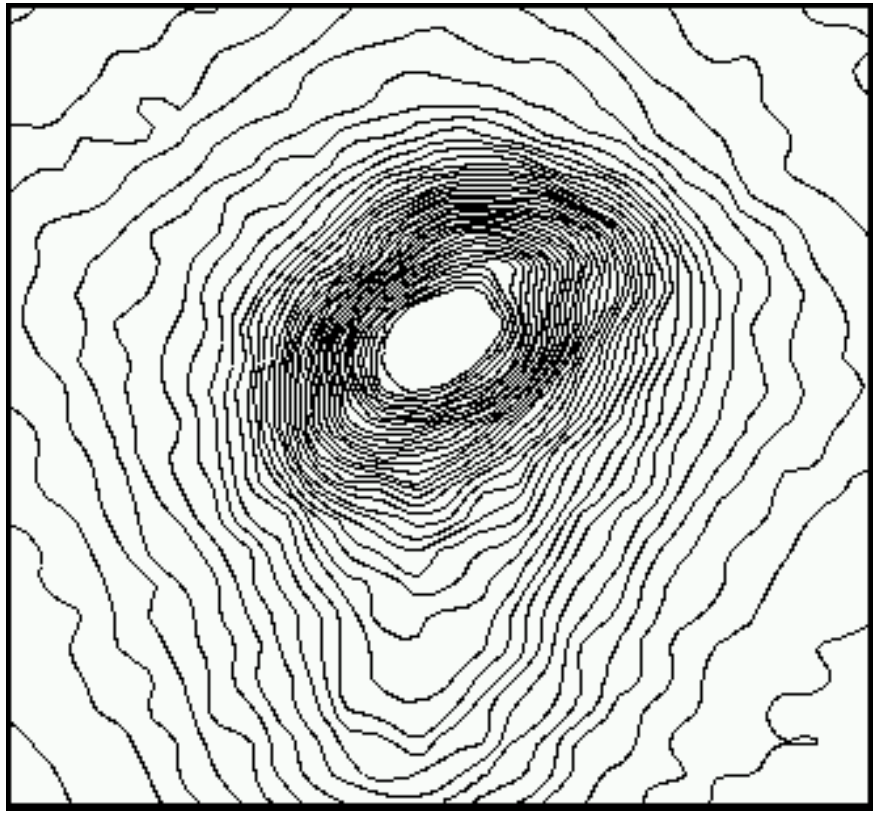

Fig. 4. Contour plot of the low excitation PN IRAS 17395-0841 in $\mathrm{H} \alpha$.

Vijapurkar et al. (1998), found $\mathrm{H} \beta, \mathrm{H} \gamma$ and several FeII and [FeII] lines in emission. Parthasarathy et al. (2000a) also classified it as a Be star. We have obtained the spectrum of this star from 5300 to $8800 \AA$ with $5 \AA$ pixel $^{-1}$ resolution at the $1.02 \mathrm{~m}$ VBO telescope (Fig. 5a). We find strong $\mathrm{H} \alpha$ emission alongwith [OI], FeII, [FeII], TiII and [TiII]. The spectral features observed appear to be very similar to the hot central star of the proto-planetary nebula HD 51585 (Klutz \& Swings 1977; Jascheck et al. 1996). The spectral energy distribution reveals the presence of warm dust at a temperature of $1200 \mathrm{~K}$. The warm dust may be a signature of ongoing post-AGB mass-loss in this star. This object was also detected in the A, C, D and E MSX bands. The narrow band CCD images also revealed $\mathrm{H} \alpha$ emission in this object. Owing to the limitations of the DUSTY code, the warm and cold dust shells were modelled independent of each other and hence the derived values of $r 1, \theta$ and $\Delta t$ may not be too appropriate.

We examined the IRAS low-resolution spectrum (LRS) of the object (Fig. 5b) retrieved from http://www.iras. ucalgary.ca/iras.html (cf. Kwok et al. 1997). The correction of the absolute calibration as in Volk \& Cohen (1989) and Cohen et al. (1992) has not been applied to the data values. While the relative shape of the LRS spectrum is OK, the absolute flux level need not match the IRAS 12 micron flux density from the photometry. The $9.7 \mu$ silicate dust feature was found to be in emission. The 8.2, 8.6 and 11.3 $\mu$ PAH features also appear to be in emission. The $9.7 \mu$ feature is expected to be in emission when the optical depth, $\tau$, of the dust envelope at $9.7 \mu$ is less than $\sim 4$. The peak strength of the feature occurs at $\tau \sim 2$. This feature originates from the circumstellar envelopes of oxygen-rich AGB stars. Modelling of the circumstellar dust using DUSTY revealed the presence of graphite grains in the warm dust close to the central star and silicate grains in the cold circumstellar dust. The combination of C-rich and O-rich features in the circumstellar environment of the star may suggest a recent change to a $\mathrm{C}$-rich chemistry of the outer envelope.

\section{\#IRAS19127+1717(=SS438)}

Whitelock \& Menzies (1986) discussed the nature of this peculiar object. They found that the object's spectrum has the characteristics of a high-density, moderate excitation PN superimposed on the continuum of a reddened early-type star. From the $\mathrm{H} \beta, \mathrm{H} \gamma$ and $\mathrm{H} \delta$ lines, they assigned a spectral classification of B9V to the star. It is not clear whether the nebula is the result of mass-loss of a single star terminating its AGB evolution or a binary system with the outward appearance of a nebula. From the Balmer decrement they obtained $E(B-V)=1.0 \pm 0.1$. Using our observed $(B-V)$ and the intrinsic $(B-V)$ for a B9V star, we find $E(B-V)=1.14$. Using the DUSTY code we obtained a distance of $4.0 \mathrm{kpc}$ to the central star of the PN. If on the other hand, the B9V star is the visual binary companion to the hot post-AGB central star of the PN, then using $M_{\mathrm{v}}=+0.2$ for the B9V star, we obtain a distance of $0.2 \mathrm{kpc}$ to the binary system. Likkel (1989) had detected OH (1667 MHz) emission in this star. CO emission was not detected (Likkel et al. 1991). We detected the presence of a warm dust component at $1300 \mathrm{~K}$ around the star. The unusually small estimate of the dynamical age from the tip of the AGB may be due to the inability to obtain a good fit to the flux distribution using the DUSTY code and hence the uncertainity in $r 1$.

\#IRAS 19157-0247(=LSIV-0229)

This object was classified as a post-AGB star with a spectral type of B1III by Parthasarathy et al. (2000a). From its $\mathrm{H} \alpha$ image, we did not detect nebulosity around the central star. The spectral energy distribution, high galactic latitude and massloss rate suggest that it may be a low mass star in the post-AGB phase of evolution.

\section{\#IRAS 19200+3457(=StHA161)}

It was found to be an $\mathrm{H}_{\alpha}$ emission star (Stephenson 1986). Preite-Martinez (1988) classified it as a possible PN. They estimated the $\mathrm{H}_{\beta}$ flux to be $4.8 \times 10^{-12} \mathrm{erg} \mathrm{s}^{-1} \mathrm{~cm}^{-2}$ for this star. Using the modified Shlowskii method, they estimated a distance of $4.3 \mathrm{kpc}$ to the star. From the dust model for the star, we estimated a distance of $3.8 \mathrm{kpc}$. The angular radius of the envelope inner boundary suggests a low excitation compact nebula $\left(<1^{\prime \prime}\right)$.

\#IRAS 19399+2312(=LSII+2317)

It was classified as B1III by Parthasarathy et al. (2000a). Reed (1998) had obtained the $U B V$ magnitudes of this star $(U=11.00, B=11.20, V=10.42)$. We found them to be in good agreement with our $B, V$, magnitudes. The spectral energy distribution showing the presence of a cold dust component at $127 \mathrm{~K}$ around the hot central star $\left(T_{\text {eff }}=24000 \mathrm{~K}\right)$ and IRAS colors similar to PNe suggest that it may be a post-AGB star. From our short exposure $\mathrm{H} \alpha$ image (120s) we did not detect nebulosity around the central star.

\section{Discussion and conclusions}

We have studied a small sample of hot post-AGB candidates selected on the basis of their IRAS colors. The near and 


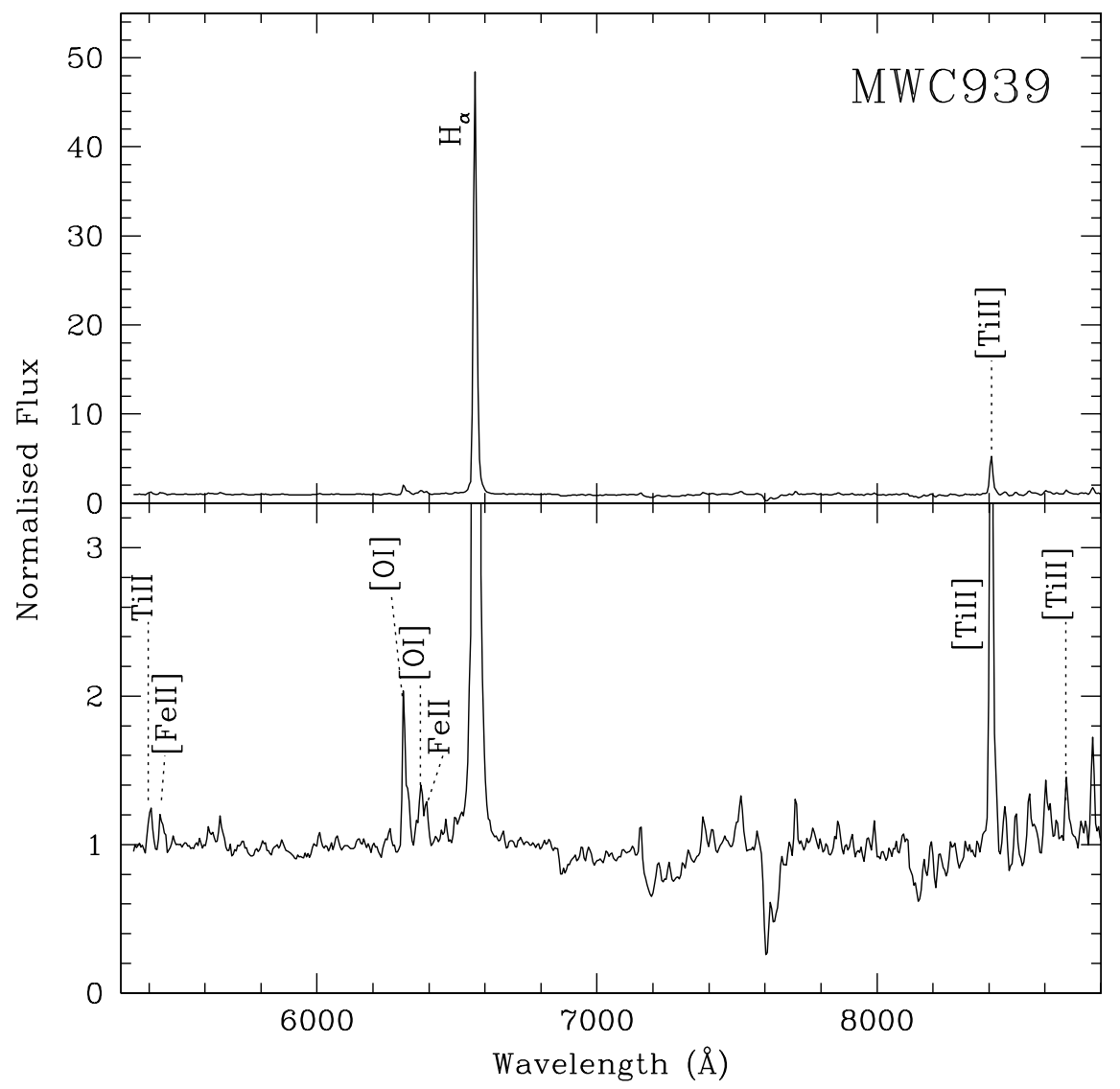

Fig. 5a. Low resolution spectrum of the hot post-AGB candidate IRAS 18313-1738 (=MWC 939).

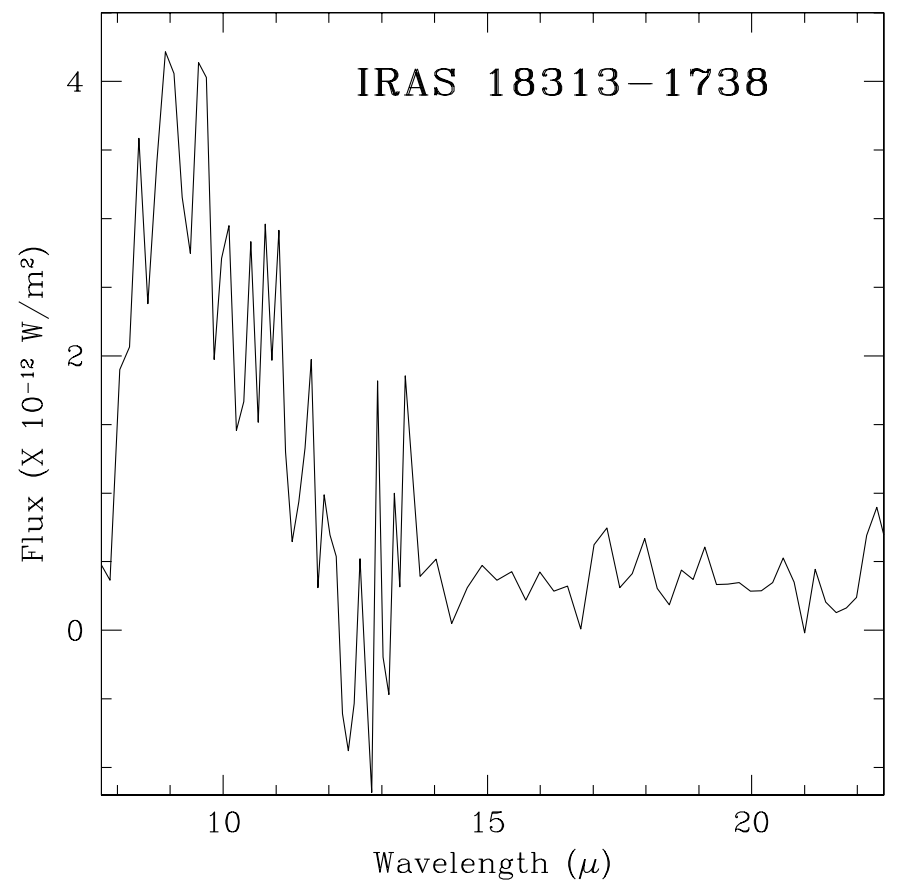

Fig. 5b. The IRAS LRS spectrum of IRAS 18313-1738 (=MWC 939).

far-infrared flux distributions of the stars were modelled using the DUSTY code. We were able to estimate the inner radii of the circumstellar shells, the distance to the stars, the mass-loss rates and angular extent of the inner boundary of the circumstellar envelopes from the best fit models. We also estimated the dynamical ages from the tip of the AGB to the present stage. However, DUSTY has certain shortcomings. It does not allow simultaneous modelling of warm and cold circumstellar dust shells. The warm and cold dust have to be modelled independent of each other and their simultaneous influence on the evolution of the nebulae cannot be accounted for. IRAS 17423-1755, IRAS 18313-1738 and IRAS 19127+1717 show the presence of both warm and cold dust in their circumstellar environment. The inner shell radii for these three objects were calculated from the model fits to the cold dust shells. Since the influence of ongoing post-AGB mass-loss (warm dust) on the remnant AGB envelope (cold dust) has not been accounted for, the derived $r 1$ values in these cases are uncertain. The uncertainity in $r 1$ translates into an uncertainity in the dynamical ages of the nebulae. Independent modelling of the warm and cold dust shells may also explain the inability to obtain a good fit in the case of IRAS 19127+1717. Besides, DUSTY allows only a spherical or slab geometry of the dust density distribution. This again may not be adequate for the hot post-AGB stars rapidly evolving into PNe of varied morphologies. In conclusion, the physical parameters derived from the modelling of the circumstellar envelopes are within the limits of the current model and may not be very precise indicators of the circumstellar envelopes of these stars.

The dust models were generated with different grain types - silicates, graphite and amorphous carbon. From the grain types for the best fit models we can infer the chemical 
composition of the circumstellar envelopes. IRAS 17074-1845 has only silicates in the circumstellar environment while the low excitation PN, IRAS 17395-0841 shows a carbon-rich circumstellar envelope. Other stars such as IRAS 17423-1755, IRAS $18313-1738$ and IRAS $19127+1717$ show a combination of silicates and carbon in the circumstellar environment. The warm dust in these stars, is composed of amorphous carbon or graphite dust grains. Since the warm dust indicates ongoing post-AGB mass-loss, the envelope chemistry appears to change from oxygen-rich to carbon-rich as the stars evolve towards the PN stage.

The stars in this paper show Balmer lines in emission. Some also show several permitted and forbidden FeII lines (e.g. MWC939) in emission and low excitation forbidden lines of SII and NII (e.g. Hen3-1475, Riera et al. 1995). Emission lines have been detected in several other hot post-AGB stars (Parthasarathy et al. 2000b). As the central stars become hotter and evolve towards the PN stage we expect to see these emission lines in their optical spectra. The objects with strong emission lines in our sample are not young stars. These objects are not associated with star forming regions. These are at high galactic latitudes with far-IR colors similar to PNe. These are most likely hot post-AGB stars and as the central stars evolve to higher temperatures they will evolve into PNe.

Acknowledgements. We would like to thank the referee for comments and suggestions that greatly helped in improving the manuscript.

\section{References}

Allen, D. A., \& Swings, J. P. 1976, A\&A, 47, 293

Beaulieu, S. F., Dopita, M. A., \& Freeman, K. C. 1999, AJ, 515, 610

Bedijn, P. J. 1987, A\&A, 186, 136

Bobrowsky, M., Zijlstra, A. A., Grebel, E. K., et al. 1995, ApJ, 446, L89

Bobrowsky, M., Sahu, K. C., Parthasarathy, M., \& García-Lario, P. 1998, Nature, 392, 469

Borkowski, K. J., \& Harrington, J. P. 2001, ApJ, 550, 778

Borkowski, K. J., Blondin, J. M., \& Harrington, J. P. 1997, ApJ, 482, L97

Blöcker, T. 1995, A\&A, 299, 755

Cohen, M., Walker, R. G., \& Witteborn, F. C. 1992, AJ, 104, 2030

Corradi, R. L. M., Schönberner, D., Steffen, M., \& Perinotto, M. 2000, A\&A, 354, 1071

Draine, B. T., \& Lee, H. M. 1984, ApJ, 285, 89

Drilling, J. S., \& Bergeron, L. E. 1995, PASP, 107, 846

Garcia-Larío, P., Manchado, A., Pych, W., \& Pottasch, S. R. 1997, A\&AS, 126, 479

Hanner, M. S. 1988, NASA Conf. Pub., 3004, 22

Henize, K. G. 1976, ApJS, 30, 491

Hog, E., Fabricius, C., Makarov, V. V., et al. 2000, A\&A, 355, 27

Iben, I., Jr., \& Renzini, A. 1983, ARA\&A, 21, 271

Ivezić, Z., Nenkova, M., \& Elitzur, M. 1999, User Manual for DUSTY, University of Kentucky Internal Report

Jacoby, G. H., Hunter, D. A., \& Christian, C. A. 1984, ApJS, 56, 257

Klutz, M., \& Swings, J. P. 1977, A\&A, 56, 143

Kwok, S., Volk, K., \& Bidelman, W. P. 1997, ApJS, 112, 557
Kwok, S. 2001, in Post-AGB Objects as a Phase of Stellar Evolution, ed. R. Szczerba, \& S. K. Górny (Boston, Dordrecht, London: Kluwer Academic Publishers), ApSS, 265, 3

Kwok, S., Su, K. Y. L., \& Stoesz, J. A. 2001, in Post-AGB Objects as a Phase of Stellar Evolution, ed. R. Szczerba, \& S. K. Górny (Boston, Dordrecht, London: Kluwer Academic Publishers), ApSS, 265, 115

Kwok, S., Volk, K., \& Hrivnak, B. J. 2002, ApJ, 573, 720

Landolt, A. U. 1992, AJ, 104, 340

Lang, K. R. 1992, Astrophysical Data: Planets and Stars (Springer-Verlag), 137

Likkel, L. 1989, ApJ, 344, 350

Likkel, L., Forveille, T., Omont, A., \& Morris, M. 1991, A\&A, 246, 153

Loup, C., Forveille, T., Nyman, L. A., \& Omont, A. 1990, A\&A, 227, L29

Massey, P., \& Davis, L. E. 1992, A User's Guide to Stellar CCD Photometry with IRAF

Mathis, J. S., Rumpl, W., \& Nordsieck, K. H. 1977, ApJ, 17, 25

Moehler, S., \& Heber, U. 1998, A\&A, 335, 985

Monet, D., Bird, A., et al. 1998, USNO-A V2.0, A Catalog of Astrometric Standards

Ossenkopf, V., Henning, Th., \& Mathis, J. S. 1992, A\&A, 261, 567

Pègouriè, B. 1988, A\&A, 194, 335

Parthasarathy, M., \& Pottasch, S. R. 1986, A\&A, 154, 216

Parthasarathy, M., \& Pottasch, S. R. 1989, A\&A, 225, 521

Parthasarathy, M. 1993a, ApJ, 414, L109

Parthasarathy, M. 1993b, in Luminous High-Latitude Stars, ASP Conf. Ser., 45, 173

Parthasarathy, M., Vijapurkar, J., \& Drilling, J. S. 2000a, A\&AS, 145, 269

Parthasarathy, M., García-Lario, P., Sivarani, T., et al. 2000b, A\&A, 357,241

Parthasarathy, M., García-Lario, P., Gauba, G., et al. 2001, A\&A, 376, 941

Pottasch, S. R., Bignell, C., Olling, R., \& Zijlstra, A. A. 1988, A\&A, 205, 248

Preite-Martinez, A. 1988, A\&AS, 76, 317

Reed, B. C. 1998, ApJS, 115, 271

Riera, A., García-Lario, P., \& Manchado, A. 1995, A\&A, 302, 137

Sanchez Contreras, C., \& Sahai, R. 2001, ApJ, 553, 173

Schlegel, D. J., Finkbeiner, D. P., \& Davis, M. 1998, ApJ, 500, 525

Schönberner, D. 1983, ApJ, 272, 708

Su, K. Y. L., Hrivnak, B. J., \& Kwok, S. 2001, AJ, 122, 1525

Szczerba, R., Górny, S. K., \& Zalfresso-Jundzillo, M. 2001, in Post-AGB Objects as a Phase of Stellar Evolution, ed. R. Szczerba, \& S. K. Górny (Boston, Dordrecht, London: Kluwer Academic Publishers), ApSS, 265, 13

Stephenson, C. B. 1986, ApJ, 300, 779

van der Veen, W. E. C. J., \& Habing, H. J. 1988, A\&A, 194, 125

Vijapurkar, J., Drilling, J. S., \& Parthasarathy, M. 1997, AJ, 114, 1573

Vijapurkar, J., Parthasarathy, M., \& Drilling, J. S. 1998, BASI, 26, 497

Volk, K., \& Cohen, M. 1989, AJ, 981918

Volk, K. M., \& Kwok, S. 1989, ApJ, 342, 345

Whitelock, P. A., \& Menzies, J. W. 1986, MNRAS, 223, 497

Whittet, D. C. B. 2003, Dust in the Galactic Environment, Second Edition (Institute of Physics Publishing), 226

Wood, P. R., \& Zarro, D. M. 1981, ApJ, 247, 247

Zijlstra, A. A., Loup, C., Waters, L. B. F. M., \& de Jong, T. 1992, A\&A, 265, L5 